\title{
Phosphorylation of chicken protein tyrosine phosphatase 1 by casein kinase II in vitro
}

\author{
Eun Joo Jung, ${ }^{1}$ Kee Ryeon $\mathrm{Kang}^{1}$ and \\ Yoon-Se Kang ${ }^{1,2}$ \\ 1 Department of Biochemistry and Gyeongsang Institute of Cancer \\ Research, College of Medicine, Gyeongsang National University, \\ Chinju 660-280, Korea \\ 2 Corresponding author
}

Accepted 17 November 1997

Abbreviations: CPTP, chicken protein tyrosine phosphatase; HPTP1B, human placenta protein tyrosine phosphatase 1B; CKII, casein kinase II; MAP kinase, mitogen-activated protein kinase; GST, glutathione S-transferase; pNPP, p-nitrophenyl phosphate; EGF, epidermal growth factor

\begin{abstract}
The phosphorylation and dephosphorylation of proteins on tyrosyl residues are key regulatory mechanisms of cell growth and signal transduction and are controlled by opposing activities of protein tyrosine kinases and phosphotyrosyl phosphatases (PTPs). We have previously cloned and characterized a nontransmembrane chicken protein tyrosine phosphatase 1 (CPTP1) similar to human placental PTP1B (HPTP1B). CPTP1 contains several phosphorylation sequence motifs (S/T-X-X-D/E) for casein kinase II (CKII), [(I>E>V)-Y-(E>G)-(E>D>P>N)-(I/V>L)] for $\mathrm{p} 56^{\text {lck}}$, and (P-E-S-P) for MAP kinase. To examine whether phosphatase activity of CPTP1 could be controlled by phosphorylation, CPTP1 and HPTP1B fusion proteins purified from $E$. coli were subjected to the in vitro phosphorylation by CKII. Phosphoamino acid analysis revealed that CPTP1 was phosphorylated on both serine and threonine residues by CKII in vitro. In addition, the degree of the phosphorylation of CPTP1 by CKII was shown to be five times higher than that of HPTP1B. Phosphorylation on both serine and threonine residues of CPTP1 in vitro results in an inhibition of its phosphatase activity. This result suggests that phosphorylation of CPTP1 and HPTP1B by CKII might be implicated in the regulation of their catalytic activities in the cell.
\end{abstract}

Keywords: chicken protein tyrosine phosphatase 1, casein kinase II

\section{Introduction}

The phosphorylation of protein tyrosine residues is an early event in signal transduction initiated by binding of growth factors and hormones to their cognate receptors and it leads to regulation of cellular activities which include proliferation, differentiation, and also malignant transformation of cells (Hunter, 1989; Ullirich and Schlessinger, 1990; Cantley et al., 1991). Under normal conditions, the level of tyrosine phosphorylation within a cell is determined by a balance between the actions of protein tyrosine kinases (PTKs) and protein tyrosine phosphatases (PTPs) (Hunter, 1989; Fischer et al., 1991; Trowbridge, 1991). PTPs do not simply reverse the action of tyrosine kinases, but rather, PTP itself may play a central role in cellular regulation. PTPs are generally classified as transmembrane (receptor-type) and nontransmembrane (nonreceptor-type) enzymes based on the presence or absence of extracellular and transmembrane portions of their predicted sequence (Fischer et al., 1991). Because the activity of tyrosine kinase can be controlled by phosphorylation, it has been postulated that PTP activity may be regulated by phosphorylation as well. For example, protein tyrosine phosphatase activity of CD45, a transmembrane PTP, is activated by sequential phosphorylation by $\mathrm{v}-\mathrm{ab}$ and casein kinase II (CKII) in vitro (Stover and Walsh, 1994). CD45 activates the src family protein-tyrosine kinases, p56 ${ }^{\text {ck }}$ and p59fyn, by dephosphorylating a negative regulatory tyrosine in the carboxyl terminus (McFarland and Thomas, 1995). A direct interaction between p56 lck and CD45 has been demonstrated upon phosphorylation of CD45 by p50csk in vitro (Autero et al., 1994).

HPTP1B, a nontransmembrane PTP, is phosphorylated on serine residue(s) in growing HeLa cells (Frangioni et al., 1992). In vitro, HPTP1B is phosphorylated at Ser386 by the mitotic protein serine/threonine kinase $\mathrm{p} 34^{\text {cdc2 }}$, and also phosphorylated at Ser-378 by protein kinase C (PKC) (Flint et al., 1993). These findings have strengthened our view that PTPs play important regulatory roles in, various cellular signaling pathways, and the activity of PTPs are regulated by phosphorylation.

To investigate possible mechanisms regulating CPTP1 protein activity, we searched for potential kinase target sites in its primary sequence. CPTP1 contains five CKII phosphorylation sequence motifs (S/T-X-X-D/E), one p56 ${ }^{\text {ck }}$ site $[(I>E>V)-Y-(E>G)-(E>D>P>N)-(I / V>L)]$, and one MAP kinase site (P-E-S-P) (Kim et al., 1996). Until now, little is known about the regulation of PTP by CKIImediated phosphorylation. In this study we demonstrated that CPTP1 is phosphorylated by CKII in vitro and this phosphorylation causes a decrease in the CPTP1 
phosphatase activity.

\section{Materials and Methods}

\section{Materials}

Casein kinase II (CKII), reduced glutathione, T4 DNA ligase, calf intestinal alkaline phosphatase (CIAP), Klenow enzyme, and most of all restriction enzymes were purchased from Boehringer Mannheim (Mannheim, Germany). Yeast extract, trypton, and bacto-agar were from Difco (Detroit, MI). [ $\gamma^{32}$ P]ATP and Kodak X-ray film were from Amersham (Buckinghamshire, UK). S-hexylglutathione agarose beads and general chemicals were from Sigma (St. Louis, MO).

\section{Bacterial expression and purification of CPTP and HPTP1B fusion proteins}

For generation of GST-CPTP, CPTP1 cDNA was digested with EcoRI and $B g / I I$ and filled with the Klenow enzyme. This CPTP DNA fragment, deleted in 72 amino acids in C-terminal side of CPTP1 coding for 356 amino acids, was ligated to the Smal-digested $p G E X-1 \mathrm{~N}$ vector ( $p G E X 1 N$ CPTP). For generation of GST-HPTP1B, HPTP1B cDNA fragment coding for amino acids 1-321 of HPTP1B was ligated to the Smal-digested pGEX-2T vector (pGEX2THPTP1B). The resulting pGEX1N-CPTP and pGEX2THPTP1B plasmids were expressed by induction with $1 \mathrm{mM}$ IPTG and purified by affinity chromatography using $S$ hexylglutathione agarose beads as described previously (Kim et al., 1996).

\section{In vitro phosphorylation}

The phosphorylation reaction of CPTP and HPTP1B fusion proteins was carried out at $30^{\circ} \mathrm{C}$ for $10 \mathrm{~min}$ by CKII in a total reaction volume of $20 \mu$ containing kinase buffer [50 mM Tris (pH 7.5), $150 \mathrm{mM} \mathrm{NaCl}, 10 \mathrm{mM} \mathrm{MgCl}_{2}$, and $10 \mathrm{mM}$ DTT], $1 \mathrm{mM}$ sodium vanadate and $5 \mathrm{mCi}$ of $\left[\gamma^{32} \mathrm{P}\right] \mathrm{ATP}$. For time course phosphorylation, CPTP and HPTP1B were reacted for 1, 5, 10, 30, and $60 \mathrm{~min}$. The reaction was terminated by mixing with SDS sample buffer, boiled, and run on a $12 \%$ SDS-PAGE. The gel was stained in staining solution $(0.125 \%$ Coomassie brilliant blue R-250/40\% methanol/10\% glacial acetic acid) and destained in a solution of $40 \%$ methanol $/ 10 \%$ glacial acetic acid. After drying, the gel was exposed to a film (Kodak x-ray) to visualize phosphorylated proteins.

\section{Phosphoamino acid analysis}

${ }^{32} \mathrm{P}$-labeled CPTP1 was prepared by in vitro phosphorylation as described above and then separated by SDSPAGE. Transfer to the PVDF membrane was carried out using Tris-glycine buffer (25 mM Tris/192 mM glycine/ $0.1 \% \mathrm{SDS} / 20 \%$ methanol), and protein was visualized by staining with Coomassie blue R-250 (0.1\% in 50\% methanol) and destained several times with destaining solution ( $50 \%$ methanol $/ 10 \%$ glacial acetic acid). The membrane was rinsed with several changes of $\mathrm{H}_{2} \mathrm{O}$, and then dried on Whatman $3 \mathrm{MM}$ filter paper. ${ }^{32} \mathrm{P}$-labeled CPTP1 band was excised, transferred to corkscrew tube, and hydrolyzed at $110^{\circ} \mathrm{C}$ for $2 \mathrm{~h}$ in $6 \mathrm{~N} \mathrm{HCl}$ under nitrogen gas. Membranes were mixed with $100 \mathrm{ml}$ methanol and $1 \mathrm{ml} \mathrm{H}_{2} \mathrm{O}$ and allowed to soak for $30 \mathrm{~min}$. The aqueous hydrolysate was lyophilized in a SpeedVac concentrator, dissolved in $10 \mathrm{ml}$ thin layer chromatography (TLC) solvent (glacial acetic acid: pyridine: $n$-butanol: $\mathrm{H}_{2} \mathrm{O}=15: 50: 75$ : 60 ), and resolved by TLC. Three $\mu \mathrm{g}$ each of phosphoserine, phosphothreonine and phosphotyrosine were used as a TLC standard marker. After fluorography with ninhydrin solution, phosphorylated residue(s) was identified by autoradiography.

\section{Determination of the tyrosine phosphatase activity}

To investigate the regulation of the phosphatase activity of CPTP1 by CKII-mediated phosphorylation, GST-CPTP was phosphorylated at $30^{\circ} \mathrm{C}$ for $30 \mathrm{~s}$ to $10 \mathrm{~min}$ with or without CKII. The phosphatase reaction was performed at $37^{\circ} \mathrm{C}$ for $10 \mathrm{~min}$ in a total reaction mixture of $100 \mu \mathrm{l}$ containing $0.1 \mathrm{M} p$-nitrophenyl phosphate (pNPP), 0.1 $\mathrm{M}$ Tris (pH 8.0), $0.25 \mathrm{M} \mathrm{NaCl}, 5 \mathrm{mM}$ EDTA and $10 \mathrm{mM}$ glutathione (Dunphy et al., 1991). At the end of the incubation, the reaction was terminated by the addition of $200 \mu \mathrm{l}$ of $0.1 \mathrm{M} \mathrm{NaOH}$. Absorbance of reaction mixture at $410 \mathrm{~nm}$ was measured in spectrophotometer with a microvolume cell.

\section{Results and Discussion}

We have previously cloned and characterized a nontransmembrane chicken protein tyrosine phosphatase (CPTP1) similar to HPTP1B (Kim et al., 1996). CPTP1 contains multiple potential phosphorylation sequence motifs for CKII, p56 ${ }^{l c k}$, and MAP kinase. This suggests that CPTP1 and HPTP1B could be phosphorylated on serine and threonine as well as tyrosine by these kinases.

Bacterial expression vectors for generation of GSTCPTP and GST-HPTP1B fusion proteins were constructed as described in "Materials and Methods". The resulting pGEX1N-CPTP and pGEX2T-HPTP1B plasmids were expressed with IPTG induction in E. coli (Figure 1, lanes 2 and 3 ) and purified by affinity chromatography using glutathione agarose beads (Figure 1, lanes 5 and 6). Additionally, GST protein as a control was expressed and purified from pGEX-2T vector (Figure 1, lanes 1 and 4).

To examine whether phosphatase activity of CPTP1 could be controlled by phosphorylation, CPTP1 and HPTP1B fusion proteins purified from $E$. coli were subjected to the in vitro phosphorylation by CKII. As shown in Figure 2, both CPTP1 and HPTP1B were phosphorylated by 


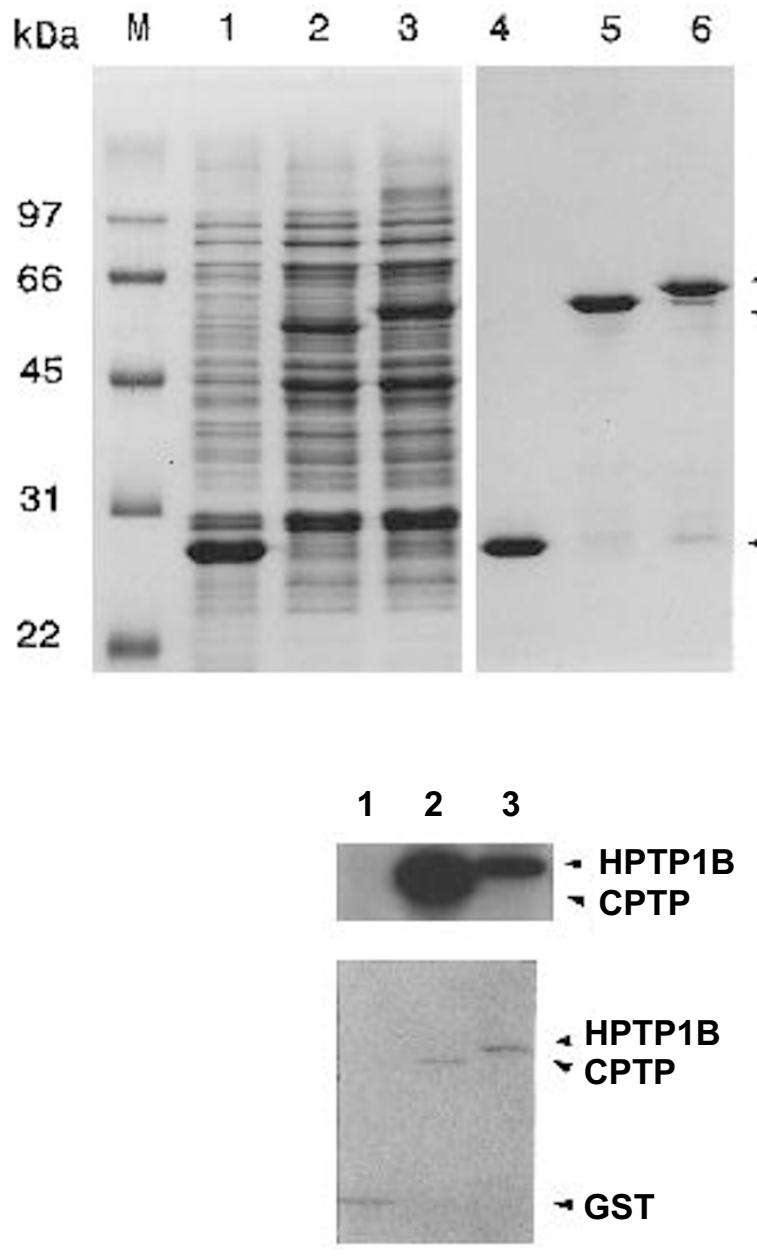

Figure 2. Phosphorylation of CPTP1 and HPTP1B by CKII in vitro. To investigate the in vitro phosphorylation of CPTP1 and HPTP1B by CKII, the phosphorylation reaction of GST-CPTP and GST-HPTP1B ( $200 \mathrm{ng}$ each) was carried out at $30^{\circ} \mathrm{C}$ for $10 \mathrm{~min}$ in a total reaction volume of $20 \mu$ c containing kinase buffer [ $50 \mathrm{mM}$ Tris (pH 7.5), $150 \mathrm{mM}$ $\mathrm{NaCl}, 10 \mathrm{mM} \mathrm{MgCl} 2$, and $10 \mathrm{mM} \mathrm{DTT}], 1 \mathrm{mM}$ sodium vanadate and $5 \mu \mathrm{Ci}$ of $[\gamma$ ${ }^{32}$ PJATP. The reaction was terminated by mixing with SDS sample buffer, boiled, and run on a $12 \%$ SDS-PAGE. After staining and destaining, the gel was exposed to a film to obtain the autoradiography.

CKII, and the degree of the phosphorylation of CPTP1 was shown to be five times higher than that of HPTP1B. The deduced amino acid sequence of CPTP1 showed $92 \%$ sequence identity to the corresponding 321 residues of the HPTP1B. In comparison with truncated form of HPTP1B, CPTP1 lacked 13 amino acid residues in Ntermination region, while it had an additional 48 residues in C-terminal region. Unfortunately, full-length CPTP1 protein was not isolated by considerable degradation of intact CPTP1 expressed in E. coli. Thus we used the truncated CPTP protein (deleted in 72 amino acids in C-terminal side of CPTP1) for in vitro phosphorylation reaction by CKII. Figure 3 shows the amino acid sequence

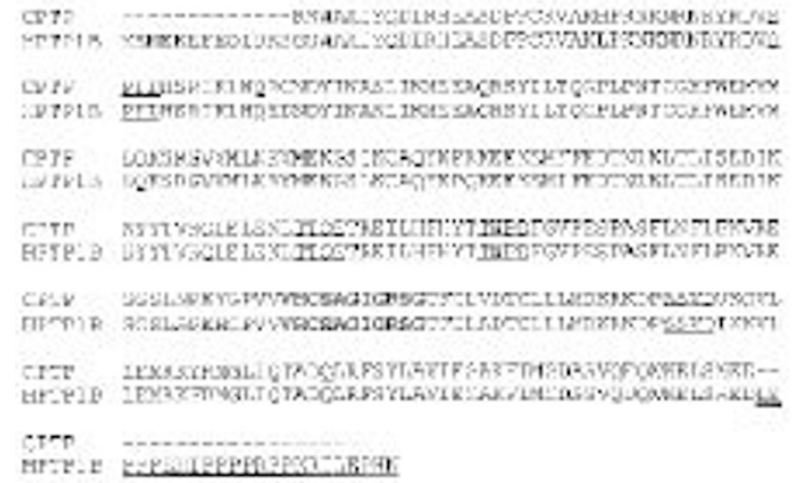

Figure 3. Amino acid sequence comparison between the truncated CPTP and HPTP1B. Four CKII phosphorylation motifs (S/T-X-X-D/E) for CPTP and HPTP1B are underlined. Conserved catalytic domain (VHCSAGIGR SG) is indicated by bold letter. The sequence marked with double underline in the $\mathrm{C}$-terminal region of HPTP1B shows the proline rich sequence.

comparison between the truncated CPTP and HPTP1B. Both CPTP and HPTP1B have four CKII phosphorylation motifs (S/T-X-X-D/E) and conserved catalytic domain of $\sim 230$ amino acid residues. Therefore, the 13 amino acid residues in $\mathrm{N}$-terminal region and the 23 amino acid proline rich sequence in C-terminal region somehow lead to the decrease in phosphorylation of HPTP1B by CKII kinase.

Figure 4 shows that CPTP1 and HPTP1B are phosphorylated in a time-dependent manner by CKII. This result suggested that four different CKII phosphorylation motifs in the CPTP protein has different reactivities with CKII enzyme. Otherwise the conformational change elicited by the one phosphorylation motif can increase 
CKII

\section{$\begin{array}{lllll}1 & 5 & 10 & 30 & 60 \mathrm{~min}\end{array}$}

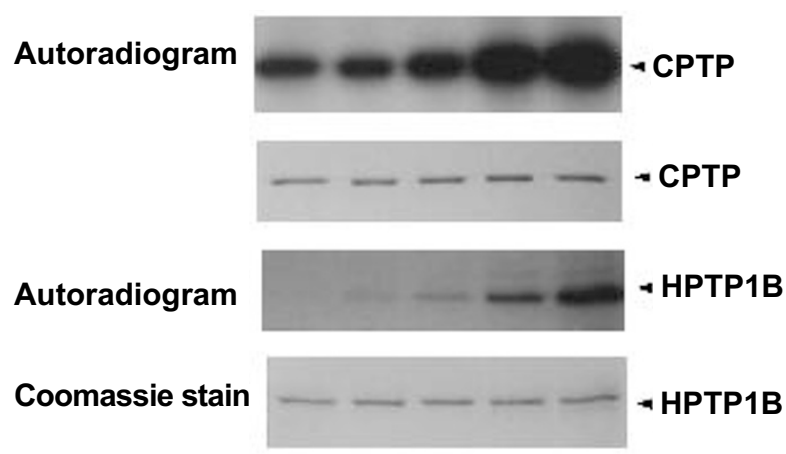

Figure 4. Time course of phosphorylation of CPTP1 and HPTP1B by CKII. The kinase reaction of GST-CPTP and GST-HPTP1B ( $300 \mathrm{ng}$ each) was carried out at $30^{\circ} \mathrm{C}$ for various times in a total reaction volume of $20 \mu \mathrm{l}$ containing kinase buffer The reaction was terminated by mixing with SDS sample buffer, boiled, and run on a $12 \%$ SDS-PAGE. After staining with Coomassie blue, the gel was dried and exposed to a X-ray film.

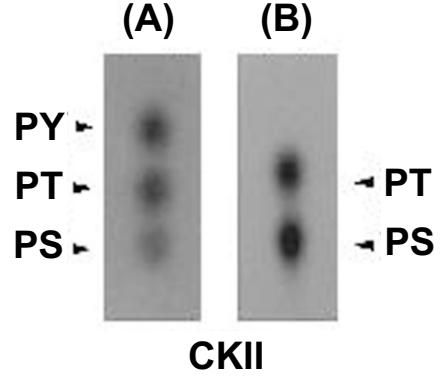

Figure 5. Phosphoamino acid analysis of CPTP1 phosphorylated by CKII. ${ }^{32} \mathrm{P}$-labeled CPTP1 by CKII was separated by SDS-PAGE, transferred to the PVDF membrane, visualized by staining with Coomassie blue $\mathrm{R}-250$, and destained several times. The membrane was rinsed with several changes of $\mathrm{H}_{2} \mathrm{O}$, and then dried on Whatman 3MM filter paper. ${ }^{32} \mathrm{P}$-labeled CPTP1 band was excised and subjected to an acid hydrolysis at $110^{\circ} \mathrm{C}$ for $2 \mathrm{~h}$ in $6 \mathrm{M} \mathrm{HCl}$, and then the aqueous hydrolysate was lyophilized and resolved by thin layer chromatography. Three $\mu \mathrm{g}$ each of phosphoserine, phosphothreonine and phosphotyrosine were used as a standard marker (A). After fluorography with ninhydrin solution, phosphorylated residues were visualized by autoradiography (B).

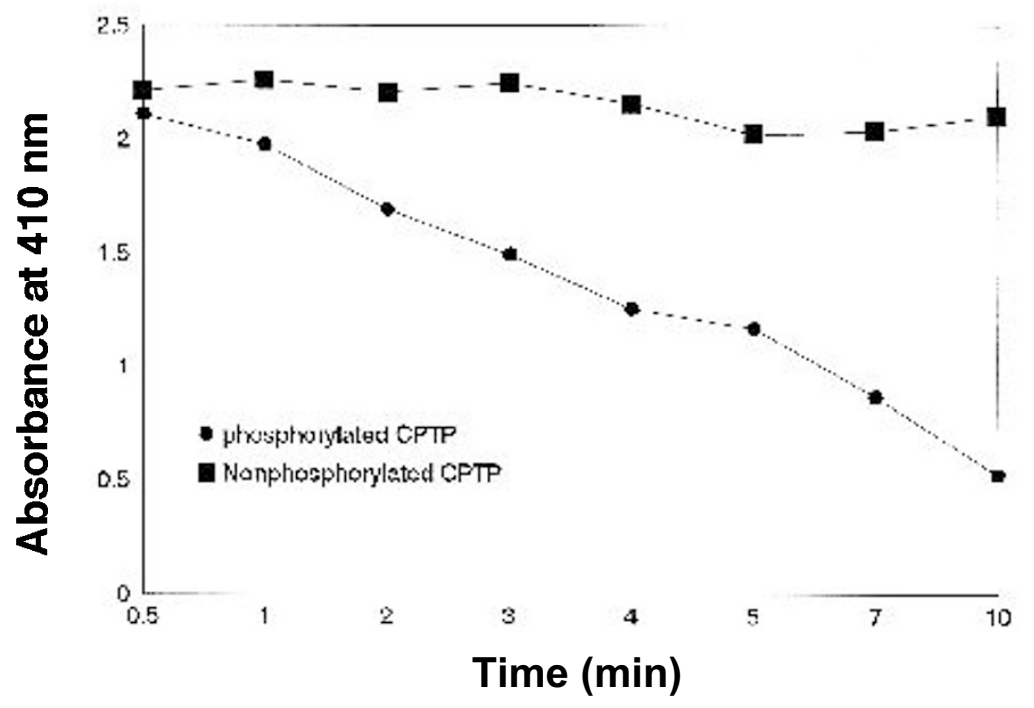

Figure 6. Regulation of the phosphatase activity of CPTP1 by CKII-mediated phosphorylation. GST-CPTP was phosphorylated at $30^{\circ} \mathrm{C}$ for $30 \mathrm{~s}$ to $10 \mathrm{~min}$ in the absence or presence of CKII. Phosphatase reaction was performed at $37^{\circ} \mathrm{C}$ for $10 \mathrm{~min}$ in a total reaction mixture of $100 \mu \mathrm{l}$ containing phosphatase buffer. The reaction was terminated by the addition of $200 \mu \mathrm{l}$ of $0.1 \mathrm{M} \mathrm{NaOH}$. Absorbance of reaction mixture was measured at $410 \mathrm{~nm}$ with a microvolume cuvette. the phosphorylation of the other motif. This sequential conformational change by initial phosphorylation might accelerate later phosphorylation of PTP when time elapses.

Phosphoamino acid analysis revealed that CPTP1 was phosphorylated on both serine and threonine residues by CKII in vitro (Figure 5). Figure 6 shows that phosphorylation of CPTP1 on these residues resulted in an inhibition of its phosphatase activity (Figure 6). In recent years, more and more proteins are reported to be phosphorylated on multiple sites in hierarchical fashion (Roach, 1991; Stover and Walsh, 1994). This multiple protein phosphorylation extends the repertoire of structural changes that might allow more intricate regulatory circuits to alter enzyme activity. Recently, the 3D-structure of HPTP1B was determined by X-ray crystallography (Barford et al., 1994; Jia et al., 1995). The catalytic site, formed from residues Cys ${ }^{215}$ to $\mathrm{Arg}^{221}$ corresponding to the conserved PTP signature motif, is located at the base of the phosphotyrosine binding pocket. Main chain nitrogens of $\mathrm{Ser}^{216}$ to $\mathrm{Arg}^{221}$ and the guanidinium side chain of $\mathrm{Arg}^{221}$ form six hydro-gen bonds and two salt bridges, respectively, with the three terminal phosphate oxygen. When CPTP1 is phos-phorylated on serine residue corresponding to $\mathrm{Ser}^{216}$ of HPTP1B by CKII, it is may change the structure of CPTP1 and inhibit enzyme activity.

Until now, there has been no report on the regulation 
of PTP by CKII-mediated phosphorylation in the cell. This paper shows that CPTP1 and HPTP1B are phosphorylated by CKII in vitro, and the phosphatase activity of CPTP1 decreases by its serine and threonine phosphorylation. This result indicates that changes in CPTP1 activity caused by phosphorylation could be implicated in its regulation by physical interaction with protein kinases.

\section{Acknowledgement}

This study was supported by the Genetic Engineering Program (1994), Ministry of Education, Republic of Korea.

\section{References}

Autero, M., Saharinen, T. P., Soula-Rothhut, M., Oetken, C., Gassmann, M., Bergman, M., Alitalo, K., Burn, P., Gahmberg, C. G. and Mustelin, T. (1994) Tyrosine phosphorylation of CD45 phosphotyrosine phosphatase by $p 50^{\text {csk }}$ kinase creates a binding site for $p 56^{l c k}$ tyrosine kinase and activates the phosphatase. Mol. Cell. Biol. 14: 1308-1321

Barford, D., Flint, A. J. and Tonks, N. K. (1994) Crystal structure of human protein tyrosine phosphatase 1B. Science 263: 1397-1404

Cantley, L. C. Auger, K. R., Carpenter, C., Duckworth, B., Graziani, A., Kapeller, R. and Soltoff, S. (1991) Oncogenes and signal transduction. Cell 64: 281-302.

Dunphy, W. G. and Kumagai, A. (1991) The cdc25 protein contains an intrinsic phosphatase activity. Cell 67: 189-196

Fischer, E. H., Charbonneau, H. and Tonks, N. K. (1991) Protein tyrosine phosphatases: a diverse family of intracellular and transmembrane enzymes. Science 253: 401-406

Flint, A. J., Gebbink, M. F., Franza, B. R., Hill, D. E. and Tonks, N. K. (1993) Multi-site phosphorylation of the protein tyrosine phosphatase, PTP1B: identification of cell cycle regulated and phorbol ester stimulated sites of phosphorylation. EMBO J. 12: 19371946

Frangioni, J. V., Beahm, P. H., Shifrin, V., Jost, C. A. and Neel, B. G. (1992) The nontransmembrane tyrosine phosphatase PTP-1B localizes to the endoplasmic reticulum via its 35 amino acid C-terminal sequence. Cell 68: 545-560

Hunter, T. (1989) Protein-tyrosine phosphatases: the other side of the coin. Cell 58: 10131016

Jia, Z., Barford, D., Flint, A. J. and Tonks, N. K. (1995) Structural basis for phosphotyrosine peptide recognition by protein tyrosine phosphatase $1 B$. Science 268 , 1754-178

Kim, C. W., Jung, E. J. and Kang Y.-S. (1996) Cloning and characterization of a chicken protein tyrosine phosphatase, CPTP1. Exp. Mol. Med. 28: 207-213

McFarland, E. D. C. and Thomas M. L. (1995) CD45 protein-tyrosine phosphatase associates with the WW domain-containing protein, CD45AP, through the transmembrane region. J. Biol. Chem. 270: 28103-28107

Roach, P. J. (1991) Multisite and hierarchal protein phoshorylation. J. Biol. Chem. 266: $14139-14142$

Stover, D. R. and Walsh, K. A. (1994) Protein-tyrosine phosphatase activity of CD45 is activated by sequential phosphorylation by two kinases. Mol. Cell. Biol. 14: 5523-5532

Trowbridge, I. S. (1991) CD45. A prototype for transmembrane protein tyrosine phosphatases. J. Biol. Chem. 266: 23517-23520

Ullrich, A. and Schlessinger, J. (1990) Signal transduction by receptors with tyrosine kinase activity. Cell 61: 203-212 